\title{
Anormalidades espermáticas de Piaractus mesopotamicus após descongelamento utilizando diferentes metodologias
}

\author{
[Abnormalities of sperm after thawing Piaractus mesopotamicus using different methodologies]
}

\author{
M.S. Paulino, L.D.S. Murgas, V.O. Felizardo, R.T.F. Freitas
}

Universidade Federal de Lavras - UFLA - Lavras, MG

\begin{abstract}
RESUMO
Avaliou-se a influência de crioprotetores, temperatura e tempo de descongelamento sobre as anormalidades espermáticas de pacu (Piaractus mesopotamicus). Amostras de sêmen de quatro pacus foram coletadas e diluídas em duas soluções crioprotetoras, dimetilsulfóxido (DMSO) 10\% e metanol $10 \%$. Após o descongelamento, foram avaliadas anormalidades primárias e secundárias. Não foi verificada interação $(\mathrm{P}>0,05)$ nas anormalidades secundárias, nas anormalidades totais $\mathrm{e}$ nos espermatozoides normais nos diferentes tratamentos empregados. Nas anormalidades primárias, foi verificada interação temperatura versus tempo de descongelamento, em que a maior temperatura associada ao menor tempo, $60^{\circ} \mathrm{C}$ por $8 \mathrm{~s}$, ou a menor temperatura associada ao maior tempo de descongelamento, $40^{\circ} \mathrm{C}$ por $12 \mathrm{~s}$, proporcionaram menor $(\mathrm{P}<0,05)$ porcentagem de espermatozoides com anormalidades primárias. No processo de criopreservação do sêmen de pacu utilizando os crioprotetores DMSO ou o metanol, recomenda-se que o descongelamento seja realizado a $40^{\circ} \mathrm{C}$ por 12 segundos ou a $60^{\circ} \mathrm{C}$ por oito segundos.
\end{abstract}

Palavras-chave: peixes, criopreservação, DMSO, metanol, sêmen

\begin{abstract}
This study aimed to evaluate the influence of cryoprotectants, thawing times and temperatures on pacu (Piaractus mesopotamicus) sperm abnormalities. Semen samples from four pacu were collected and diluted in 2 solutions, cryoprotectant dimethylsulfoxide (DMSO) 10\% and methanol 10\%. After thawing, semen was collected for morphological analysis, evaluating primary and secondary abnormalities. There was no interaction $(P>0.05)$ of secondary abnormalities, abnormalities in total and normal sperm in the different treatments. The primary abnormalities showed interaction between temperature and thawing time, where the higher temperature was associated with the shortest time $\left(60^{\circ} \mathrm{C}\right.$ for $\left.8 \mathrm{~s}\right)$ or the lowest temperature associated with longer thawing $\left(40^{\circ} \mathrm{C}\right.$ for $\left.12 \mathrm{~s}\right)$ provided under $(P<0.05)$ percentages of spermatozoa with primary abnormalities. In the process of semen cryopreservation of $P$. mesopotamicus using the cryoprotectants DMSO and methanol, it is recommended that thawing be performed at $40^{\circ} \mathrm{C}$ for 12 seconds or $60^{\circ} \mathrm{C}$ for 8 seconds.
\end{abstract}

Keywords: fish, cryopreservation, DMSO, methanol, semen

\section{INTRODUÇÃO}

Devido à expansão da piscicultura nos últimos anos, várias técnicas têm se desenvolvido no intuito de preservar gametas (Carolsfeld et al., 2003). O processo de criopreservação de sêmen de peixes envolve várias etapas, nas quais são utilizadas soluções crioprotetoras adequadas, ativadores que possam promover e prolongar a capacidade natatória dos espermatozoides e o descongelamento (Murgas et al., 2001). No entanto, ao longo do processo de criopreservação, danos podem ocorrer nas células espermáticas (Medeiros et al., 2002).

Recebido em 11 de agosto de 2011

Aceito em 20 de julho de 2012

E-mail:mspmichelle@bol.com.br 
Entre as características para verificar a qualidade do sêmen, a motilidade espermática é importante para a avaliação do processo de criopreservação do sêmen de peixes (Rurangawa et al., 2004; Godinho, 2007). Alguns autores sugerem que exames morfopatológicos dos espermatozoides de peixes sejam incorporados à rotina de avaliação do sêmen (Streit et al., 2006) o que pode auxiliar na caracterização de amostras congeladas, explicando insucessos na reprodução (Yao, 2000). Algumas técnicas têm sido desenvolvidas com o objetivo de avaliar a morfologia espermática, entre elas a utilização de corantes na preparação de esfregaços. Segundo Streit Jr. et al. (2004), o corante rosa bengala mostrou-se eficiente na preparação da coloração de esfregaços do sêmen de pacu.

O pacu (Piaractus mesopotamicus) é um peixe utilizado nas pisciculturas e nos repovoamentos de reservatório. A criação dessa espécie tem sido incentivada em razão do seu alto valor comercial e da grande aceitação no mercado nacional (Vaz et al., 2000).

Os primeiros estudos envolvendo a criopreservação com sêmen de pacu limitaram-se a avaliar a motilidade progressiva (Carolsfeld et al., 2003) e a taxa de fertilização (Silveira et al., 1990). Posteriormente, Streit Jr. et al. (2009), Felizardo et al. (2010) e Miliorini et al. (2011) realizaram análise da morfologia espermática pós-congelamento de pacu, no caso do primeiro estudo, e de Prochilodus lineatus. Porém, esses autores não avaliaram a influência da temperatura e do tempo de descongelamento sobre as anormalidades morfológicas. Diante disso, este trabalho teve como objetivo avaliar a influência de crioprotetores, temperatura e tempo de descongelamento sobre as anormalidades espermáticas de pacu.

\section{MATERIAL E MÉTODOS}

O experimento foi realizado no período de novembro/2008 a fevereiro/2009. Foram utilizados quatro reprodutores provenientes do plantel da Estação Ambiental da Companhia Energética de Minas Gerais (CEMIG). Os animais aptos a receberem a indução hormonal foram selecionados mediante a verificação da liberação de sêmen sobre leve pressão da cavidade celomática, sendo encaminhados para aquários de $2000 \mathrm{~L}$ à densidade de três peixes/aquário. Os exemplares foram pesados e permaneceram em jejum por 24 horas até serem submetidos ao tratamento hormonal com $1 \mathrm{mg} / \mathrm{kg}$ de extrato bruto de hipófise de carpa (EBHC).

Após oito horas da aplicação hormonal, o peixe foi capturado com um puçá e envolto em uma toalha, sendo totalmente seco para evitar a ativação do sêmen com água. A coleta do sêmen foi realizada em tubo de ensaio, e este foi encaminhado para avaliação qualitativa. Em lâmina focalizada a microscópio óptico, o sêmen foi ativado com água destilada na proporção de 1:4 sêmen:água, sendo avaliada subjetivamente a taxa de motilidade espermática. Todas as amostras de sêmen analisadas apresentaram taxas de motilidade espermática acima de $80 \%$.

Para o processo de congelamento, o sêmen de cada animal foi diluído em duas diferentes soluções crioprotetoras [metanol 10\% + Beltisville Thawing Solution (BTS) $5 \%$ e dimetilsulfóxido (DMSO) $10 \%$ + BTS 5\%], em uma proporção de 1:4 sêmen:solução crioprotetora (Miliorini et al., 2011). O sêmen diluído foi envasado em palhetas de $0,5 \mathrm{~mL}$, e estas foram imediatamente acondicionadas em raques de polietileno e colocadas em botijão de vapor de nitrogênio (Taylor-Warton, modelo CP 300, dry shipper). Após 24 horas, as raques foram transferidas para um botijão contendo nitrogênio líquido (Cryometal, modelo DS-18), onde permaneceram por três dias. As palhetas contendo $\mathrm{o}$ sêmen criopreservado foram descongeladas em temperaturas de $60{ }^{\circ} \mathrm{C}$ e $40{ }^{\circ} \mathrm{C}$ por oito e 12 segundos, originando, dessa forma, oito tratamentos (Tab. 1).

Para análise das anormalidades espermáticas de cada animal, $10 \mu \mathrm{L}$ de sêmen descongelado nas diferentes temperaturas e tempos foram diluídos em $990 \mu \mathrm{L}$ de solução fixadora de formol-citrato. A diluição do sêmen in natura foi utilizada como controle para a avaliação das anormalidades totais. Dessa diluição, uma alíquota de sêmen submetido foi depositada em lâmina histológica, corada com rosa bengala e levada ao microscópio óptico com objetiva de 100X, onde foram avaliados 200 espermatozoides por lâmina (Streit Jr. et al., 2004).

Foram verificadas anormalidades primárias: macrocefalia, microcefalia, cabeça degenerada, peça intermediária degenerada (PID), cauda 
fraturada, cauda fortemente enrolada e cauda degenerada; e anormalidades secundárias: cabeça isolada, cauda apresentando gota citoplasmática proximal, gota citoplasmática distal e cauda dobrada (Miliorini et al., 2011; Paulino et al., 2011).

O delineamento experimental foi em blocos ao acaso, em um esquema fatorial $2 \times 2 \times 2$ (dois crioprotetores $\mathrm{x}$ duas temperaturas $\mathrm{x}$ dois tempos de descongelamento). Cada animal representou o bloco, perfazendo-se um total de quatro animais, sendo estes considerados como unidade experimental. Para análise dos dados, foi utilizado o programa SAEG (Sistema para análises estatísticas, 2007), e as médias foram comparadas utilizando-se o teste de Scott- Knott a $5 \%$ de probabilidade.

Tabela 1. Descrição dos tratamentos empregados para avaliação morfológica do sêmen de pacu

\begin{tabular}{|c|c|c|c|}
\hline & Crioprotetor & Temperatura $\left({ }^{\circ} \mathrm{C}\right)$ & Tempo de descongelamento (s) \\
\hline 1 & \multirow{4}{*}{ DMSO } & \multirow{2}{*}{60} & 8 \\
\hline 2 & & & 12 \\
\hline 3 & & \multirow{2}{*}{40} & 8 \\
\hline 4 & & & 12 \\
\hline 5 & \multirow{4}{*}{ Metanol } & \multirow{2}{*}{60} & 8 \\
\hline 6 & & & 12 \\
\hline 7 & & \multirow{2}{*}{40} & 8 \\
\hline 8 & & & 12 \\
\hline
\end{tabular}

\section{RESULTADOS}

Não foi verificada interação significativa $(\mathrm{P}>0,05)$ nas anormalidades secundárias, anormalidades totais e entre os espermatozoides normais nos diferentes tratamentos empregados no sêmen de pacu (Tab. 2), isto é, não houve interação do crioprotetor versus temperaturas versus tempo de descongelamento, no entanto, as anormalidades primárias apresentaram interação $\mathrm{da}$ temperatura versus tempo de descongelamento, em que a maior temperatura associada ao menor tempo $\left(60^{\circ} \mathrm{C}\right.$ por $\left.8 \mathrm{~s}\right)$ ou a menor temperatura associada ao maior tempo de descongelamento $\left(40^{\circ} \mathrm{C}\right.$ por $\left.12 \mathrm{~s}\right)$ proporcionaram menores $\quad(\mathrm{P}<0,05) \quad$ porcentagens de espermatozoides com anormalidades primárias (Tab. 2).

A porcentagem de anormalidades totais observadas no sêmen de pacu nos diferentes tratamentos empregados foi mais alta $(\mathrm{P}<0,05)$ que a no sêmen in natura (Tab. 2).

Tabela 2. Porcentagem de anormalidades primárias e secundárias verificadas em espermatozoides de pacu

\begin{tabular}{|c|c|c|c|c|}
\hline Tratamento & Anorm. prim. $(\%)^{*}$ & $\begin{array}{c}\text { Anorm. sec. } \\
(\%)\end{array}$ & $\begin{array}{c}\text { Anorm. total } \\
(\%)^{* *}\end{array}$ & $\begin{array}{c}\text { Normal } \\
(\%)\end{array}$ \\
\hline 1 & $6,0 \pm 2,6$ & $14,4 \pm 11,8$ & $23,4 \pm 13,1$ & $54,3 \pm 26,2$ \\
\hline 2 & $7,0 \pm 2,4$ & $14,8 \pm 11,6$ & $19,8 \pm 9,6$ & $58,5 \pm 19,7$ \\
\hline 3 & $9,0 \pm 2,8$ & $13,2 \pm 7,4$ & $19,3 \pm 5,6$ & $57,5 \pm 11,3$ \\
\hline 4 & $5,9 \pm 2,9$ & $8,4 \pm 1,9$ & $15,2 \pm 3,9$ & $71,0 \pm 7,8$ \\
\hline 5 & $6,2 \pm 4,8$ & $20,1 \pm 3,9$ & $29,4 \pm 4,6$ & $43,3 \pm 9,3$ \\
\hline 6 & $9,0 \pm 3,9$ & $19,4 \pm 4,2$ & $26,4 \pm 2,8$ & $45,3 \pm 5,7$ \\
\hline 7 & $8,1 \pm 4,6$ & $8,1 \pm 3,8$ & $24,4 \pm 11,5$ & $51,3 \pm 23,0$ \\
\hline 8 & $5,1 \pm 4,9$ & $9,6 \pm 2,3$ & $20,8 \pm 7,7$ & $64,5 \pm 7,6$ \\
\hline In natura $(\%)$ & & & $9,2 \pm 0,9$ & \\
\hline
\end{tabular}


Dentro das anormalidades primárias, a utilização do crioprotetor DMSO (tratamentos 1, 2, 3 e 4) proporcionou menor porcentagem de anormalidades da peça intermediária (Tab. 3) em relação à utilização do metanol. Porém, não foram verificadas diferenças significativas em anormalidades de flagelo e cabeça nos diferentes tratamentos empregados.

Nas anormalidades secundárias, não foram verificadas diferenças significativas $(\mathrm{P}>0,05)$ entre as características avaliadas nos diferentes tratamentos empregados (Tab. 4).

Tabela 3. Porcentagem de anormalidades do flagelo, cabeça e peça intermediária dentro das anormalidades primárias

\begin{tabular}{cccc}
\hline \multirow{2}{*}{ Tratamento } & \multicolumn{3}{c}{ Anormalidades primárias (\%) } \\
\cline { 2 - 4 } & Flagelo & Cabeça & PI * \\
\hline 1 & $86,2 \pm 19,6$ & $8,2 \pm 10,6$ & $5,5 \pm 9,1$ \\
3 & $67,6 \pm 17,8$ & $27,6 \pm 23,9$ & $4,6 \pm 9,3$ \\
4 & $78,6 \pm 14,3$ & $21,3 \pm 14,3$ & $0,0 \pm 0,0$ \\
5 & $72,2 \pm 18,7$ & $20,6 \pm 19,9$ & $7,0 \pm 10,7$ \\
6 & $73,8 \pm 16,6$ & $11,4 \pm 9,9$ & $14,7 \pm 39,6$ \\
7 & $63,6 \pm 8,1$ & $19,5 \pm 19,5$ & $16,8 \pm 12,6$ \\
8 & $69,7 \pm 25,0$ & $19,4 \pm 16,3$ & $10,8 \pm 11,6$ \\
& $74,2 \pm 21,9$ & $11,1 \pm 7,4$ & $14,6 \pm 16,9$ \\
\hline
\end{tabular}

PI. peça intermediária. 1-DMSO,60 $0^{\circ}, 8 \mathrm{~s} ; 2$-DMSO, $60^{\circ}, 12 \mathrm{~s} ; 3-\mathrm{DMSO}, 40^{\circ}, 8 \mathrm{~s} ; 4-\mathrm{DMSO}, 40^{\circ}, 12 \mathrm{~s} ; 5$-metanol, $60^{\circ}, 8 \mathrm{~s} ; 6^{6-}$ metanol, $60^{\circ}, 12 \mathrm{~s} ; 7$-metanol $, 40^{\circ}, 8 \mathrm{~s} ; 8$-metanol, $40^{\circ}, 12 \mathrm{~s}$. *Diferença significativa entre os crioprotetores.

Tabela 4. Porcentagem de anormalidades do flagelo, cabeça e gota dentro das anormalidades secundárias

\begin{tabular}{cccc}
\hline \multirow{2}{*}{ Tratamento } & \multicolumn{3}{c}{ Anormalidades secundárias } \\
\cline { 2 - 4 } & Flagelo & Cabeça & Gota \\
\cline { 2 - 4 } 1 & $72,3 \pm 17,8$ & $27,6 \pm 17,8$ & $0,0 \pm 0,0$ \\
2 & $91,6 \pm 9,7$ & $8,3 \pm 9,7$ & $0,0 \pm 0,0$ \\
3 & $64,6 \pm 18,0$ & $34,1 \pm 19,5$ & $1,2 \pm 2,5$ \\
4 & $55,5 \pm 35,0$ & $41,1 \pm 34,5$ & $3,3 \pm 6,6$ \\
5 & $74,3 \pm 4,9$ & $25,6 \pm 4,9$ & $0,0 \pm 0,0$ \\
6 & $80,2 \pm 6,5$ & $19,7 \pm 6,5$ & $0,0 \pm 0,0$ \\
7 & $76,4 \pm 19,8$ & $11,0 \pm 11,7$ & $12,5 \pm 25,0$ \\
8 & $63,4 \pm 26,5$ & $24,0 \pm 29,7$ & $12,5 \pm 25,0$ \\
\hline
\end{tabular}

1-DMSO, $60^{\circ}, 8 \mathrm{~s} ; \quad 2-\mathrm{DMSO}, 60^{\circ}, 12 \mathrm{~s} ; \quad 3$-DMSO $, 40^{\circ}, 8 \mathrm{~s} ; 4-\mathrm{DMSO}, 40^{\circ}, 12 \mathrm{~s} ; 5$-metanol, $60^{\circ}, 8 \mathrm{~s} ; \quad 6$-metanol, $60^{\circ}, 12 \mathrm{~s} ; \quad 7$ metanol, $40^{\circ}, 8 \mathrm{~s} ; 8$-metanol, $40^{\circ}, 12 \mathrm{~s}$

\section{DISCUSSÃO}

A porcentagem de anormalidade total verificada no sêmen pós-congelamento variou de $15,2 \pm 3,9$ a $26,4 \pm 2,8$. Embora a porcentagem máxima de anormalidades espermática para utilização do sêmen no processo de fertilização não seja as determinada para peixes, o Colégio Brasileiro de Reprodução Animal recomenda não utilizar sêmen para fertilização com índices de espermatozoides com anormalidade acima de $30 \%$ em bovinos e equinos, e $20 \%$ em ovinos e suínos. Pode-se sugerir, dessa forma, que os tratamentos empregados podem ser utilizados no processo de criopreservação e descongelamento do sêmen de pacu, tendo em vista os índices de anormalidades considerados para as espécies citadas. Por outro lado, vale ressaltar que, do total das anormalidades verificadas, correspondem anormalidades secundárias que não afetam diretamente a capacidade fertilizante do espermatozoide, as quais, segundo Herman et al. (1994), estariam relacionadas aos procedimentos de manejo durante a coleta do sêmen e à confecção das lâminas para avaliação.

A influência da interação temperatura versus tempo de descongelamento sobre as anormalidades primárias pode ser atribuída, principalmente, ao aquecimento final adquirido pela paleta após sua imersão na água, tendo em vista também que, em uma menor temperatura, o sêmen pode demorar mais tempo para descongelar. Miranda et al. (2009) afirmaram 
que a temperatura exerce efeitos diretos sobre a gametogênese e sobre a liberação de gonadotropinas hipofisárias.

A porcentagem de anormalidade total verificada neste trabalho com os diferentes tratamentos empregados é mais alta que a do sêmen in natura $(9,2 \pm 0,9 \%)$, porém as anormalidades verificadas no sêmen tratado é mais baixa que a verificada por Streit Jr. et al. (2006) no sêmen in natura da mesma espécie $(41,8 \%)$. A porcentagem de anormalidades primárias verificadas nos diferentes tratamentos apresentou variação de $5 \pm 2,4$ a $11,1 \pm 4,9 \%$, menor que as anormalidades primárias verificadas no sêmen in natura por este mesmo autor. Essa diferença dentro da mesma espécie pode estar relacionada com as condições em que os animais são mantidos, pois, segundo Herman et al. (1994), as anormalidades dos espermatozoides em outros animais podem estar relacionadas com deficiência nutricional, idade dos machos, consanguinidade, além de doenças que possam acometer os reprodutores.

Porém os danos causados aos espermatozoides pelo processo de criopreservação já são relatados em espécies reofílicas como a curimba $(P$. lineatus) (Felizardo et al., 2010) e até mesmo para o próprio pacu (Streit Jr. et al., 2006). De acordo com Baulny et al. (1997), isso ocorre em problemas de integridade e alteração no protoplasma da célula após o congelamento, podendo, dessa forma, afetar a capacidade do espermatozoide em fecundar o ovócito.

Neste trabalho, não foi verificada diferença $(\mathrm{P}>0,05)$ nas anormalidades espermáticas entre os diferentes crioprotetores utilizados. Felizardo et al. (2010) também não verificaram diferença nas anormalidades totais do sêmen de pacu após congelamento, utilizando-se DMSO e metanol como crioprotetores. Miliorini et al. (2011) concluíram que DMSO 7,5\% e metanol 5\% conferem adequada proteção ao sêmen de pacu durante o processo de criopreservação, visto que essa proteção se dá sobre as enzimas lábeis e sobre a estabilidade das proteínas em soluções aquosas.

Entre as anormalidades primárias encontradas, as de peça intermediária foram mais baixas ao se utilizar DMSO como crioprotetor em relação ao uso do metanol. Este fato pode ser atribuído às características da rápida penetração intracelular que o metanol possui (Jamieson, 1991), visto que o processo de criopreservação causa desestabilização das membranas lipoproteicas das células espermáticas, sobretudo das mitocôndrias localizadas na peça intermediária.

\section{CONCLUSÕES}

No processo de criopreservação do sêmen de pacu utilizando os crioprotetores DMSO ou o metanol, recomenda-se que o descongelamento seja realizado a $40^{\circ}$ por 12 segundos ou a $60^{\circ}$ por oito segundos para que não haja alteração na porcentagem de anormalidades espermáticas, impedindo, dessa forma, que essa característica afete a fertilização.

\section{AGRADECIMENTOS}

Os autores agradecem à CEMIG, pela disponibilização do local e dos reprodutores de peixes; à FAPEMIG, à CAPES e ao CNPq, pelo apoio financeiro para realização deste projeto.

\section{REFERÊNCIAS}

BAULNY, B.O.; LE VERN, Y.; KERBOEUF, D. et al. Flow cytometric evaluation of mitochondrial activity and membrane integrity in fresh and cryopreserved rainbow trout spermatozoa. Cryobiology, v.34, p.141-149, 1997.

CAROLSFELD, J.; GODINHO, H.P.; ZANIBONI FILHO, E. et al. Cryopreservation of sperm in brazilian migratory fish conservation. J. Fish Biol., v.63, p.472-489, 2003.

FELIZARDO, V.O.; MELLO, R.A.; MURGAS, L.D.S. et al. Effect of cryopreservant combinations on the motility and morphology of curimba (Prochilodus lineatus) sperm. Anim. Reprod. Sci., v.122, p.259-263, 2010.

GODINHO, H.P. Estratégias reprodutivas de peixes aplicadas á aquicultura: bases para $\mathrm{O}$ desenvolvimento de tecnologias de produção. Rev. Bras. Reprod. Animal, v.31, p.351-360, 2007.

HERMAN, H.A.; MITCHELL, J.R.; DOAK, G.A. The artificial insemination and embryo transfer of diary and beef cattle. Illinois: Interstate Publisher, 1994. 392 p. 
JAMIESON, B.G.M. Fish evolution and systematics: evidence from spermatozoa. Cambridge: Cambridge University, 1991. 139p.

MEDEIROS, C.M.O.; FORELL, F.; OLIVEIRA, A.T.D. et al. Current status of sperm cryopreservation: why isn't it better? Theriogenology, v.57, p.327-344, 2002.

MILIORINI, A.B.; MURGAS, L.D.S.; ROSA, P.V. et al. A morphological classification proposal for curimba (Prochilodus lineatus) sperm damages after cryopreservation. Aquac. Res., v.42, p.177-187, 2011.

MIRANDA, L.A.; STRÜSSMANN, C.A.; SOMOZA, G.M. Effects of light and temperature conditions on the expression of $\mathrm{GnRH}$ and $\mathrm{GtH}$ genes and levels of plasma steroids in Odontesthes bonariensis females. Fish Physiol. Biochem., v.35, p.101-108, 2009.

MURGAS, L.D.S.; GUALHANONE, A.; SILVA, M.O.B. et al. Calidad seminal del pez piracanjuba (Brycon orbignyanus) postdescongelación. Rev. Anales Vet., v.17, p.3-10, 2001.

PAULINO, M.S.; MILIORINI, A.B.; MURGAS, L.D.S.; LIMA, F.S.M.; FELIZARDO, V.O. Desempenho reprodutivo do pacu, piracanjuba e curimba induzidos com extrato com extrato de busserelina. Bol. Inst. Pesca, v.37, p.39-45, 2011.

RURANGWA, E.; KIME, D.E.; OLLEVIER, F. et al. The measurement of sperm motility and factors affecting sperm quality in cultured fish. Aquaculture, v.234, p.1-28, 2004.
SILVEIRA, W.F.; KAVAMOTO, E.T.; CESTAROLLI, M.A. et al. Avaliação espermática, preservação criogênica e fertilidade do sêmen do pacu, Piaractus mesopotamicus (Holmberg, 1887), proveniente de reprodução induzida. Bol. Inst. Pesca, v.17, p.1-13, 1990.

SISTEMA para Análises Estatísticas-SAEG, Versão 9.1: Fundação Arthur Bernardes - UFV Viçosa, 2007.

STREIT JR., D.P.; BENITES, C.; MORAES, G.V. et al. Sêmen de pacu (Piaractus mesopotamicus) criopreservado com diluentes utilizados para sêmen de suínos. Cienc. Anim. Bras., v.7, p.289-297, 2006.

STREIT JR., D.P.; MORAES, G.V.; RIBEIRO, R.P. et al. Avaliação de diferentes técnicas para coloração de sêmen de peixes. Arq. Cienc. Vet. Zool., v.7, p.157-162, 2004.

STREIT JR., D.P.; OLIVEIRA, A.C.; RIBEIRO, R.P. et al. Motilidade, vigor e patologias seminal in natura e pós criopreservação de Piaractus mesopotamicus. Bol. Inst. Pesca, v.35, p.159167, 2009.

VAZ, M.M.; TORQUATO, V.C.; BARBOSA, N.D.C. Guia ilustrado de peixes da bacia do rio Grande. Belo Horizonte: CEMIG/CETEC, 2000. $144 \mathrm{p}$.

YAO, Z.; CRIM, L.W.; RICHARDSON, G.F. et al. Motility, fertility and ultrastructural changes of ocean pout (Macrozoarces americanus L.) sperm after cryopervation. Aquaculture, v.181, p.361-375, 2000. 